\title{
Establishment of a functional symbiosis between the cyanobacterium Nostoc punctiforme and the bryophyte Anthoceros punctatus requires genes involved in nitrogen control and initiation of heterocyst differentiation
}

\author{
Francis C. Y. Wong and John C. Meeks
}

Author for correspondence: John C. Meeks. Tel: +1 530752 3346. Fax: +1 5307529014. e-mail: jcmeeks@ucdavis.edu

Section of Microbiology, Division of Biological Sciences, University of California, One Shields Avenue, Davis, CA 95616 USA

\begin{abstract}
Three mutant strains (ntcA, hetR, hetF) of the cyanobacterium Nostoc punctiforme unable to differentiate heterocysts were characterized and examined for their ability to form a symbiotic association with the bryophyte Anthoceros punctatus. Previously unknown characteristics of the $\boldsymbol{N}$. punctiforme hetR mutant include differentiation of chilling-resistant akinetes, while vegetative cells of the ntcA mutant randomly lysed, yielding short filaments, following ammonium deprivation. Strains with mutations in hetF and hetR infected $A$. punctatus with similar frequency to that of wild-type $N$. punctiforme but did not support growth of the plant partner. These results confirm that the infection of $\boldsymbol{A}$. punctatus by hormogonia leading to the establishment of an association is physiologically uncoupled from the development of a functional diazotrophic association. They also indicate that heterocyst regulatory elements downstream from HetR and HetF are required in both free-living and symbiotic heterocyst differentiation and nitrogenase expression. A strain with a mutation in the global nitrogen regulator ntcA did not infect $A$. punctatus despite its ability to differentiate hormogonia at a low frequency. When complemented with one or more copies of ntcA, the mutant strain infected $A$. punctatus at a similar frequency as the wild-type and supported growth of the plant partner in the absence of combined nitrogen. These results established a connection between the presence of a functional copy of ntcA and the magnitude of hormogonium differentiation, and the behaviour of the formed hormogonia.
\end{abstract}

Keywords: cellular differentiation, cyanobacteria, nitrogen control, nitrogen fixation

\section{INTRODUCTION}

The oxygenic phototrophic cyanobacterium Nostoc punctiforme and related members of the unbranched filamentous order Nostocales have exceptionally diverse morphological, physiological and ecological properties (Castenholz \& Waterbury, 1989). N. punctiforme veg-

Abbreviation: HIF, hormogonium-inducing factor.

The GenBank accession numbers for the coding regions of $n t c A$, het $R$ and hetF are AY038370, AF318069 and AF288130, respectively. etative cells have three developmental alternatives in response to environmental changes. The first and most well-known developmental alternative is the patterned differentiation of about $8 \%$ of the vegetative cells into nitrogen-fixing heterocysts when combined nitrogen is limited (Wolk, 2000; Wolk et al., 1994). Second, under conditions of energy limitation such as low light intensity or phosphate starvation, vegetative cells can differentiate into spore-like akinetes for survival through unfavourable growth conditions (Adams \& Duggan, 1999). Akinetes are suggested to be the evolutionary precursors of heterocysts (Wolk et al., 1994). Third, 
following the sensing of a positive or negative stress signal for growth, all vegetative cells of a filament can divide uncoupled from biomass increase and DNA replication to form small-celled, relatively short, motile filaments called hormogonia, which lack heterocysts and function in short-distance dispersal (Tandeau de Marsac, 1994). The completion of a hormogonium developmental cycle is marked by the resumption of cellular growth and DNA replication, and by differentiation of heterocysts when nitrogen is limited.

Many Nostoc strains, including N. punctiforme, also form diazotrophic symbiotic associations with a variety of terrestrial plants (Meeks, 1998). Differentiation and behaviour of hormogonia and heterocysts are altered in the symbiotic growth state. Diazotrophic cyanobacteria-plant symbioses have similarities to associations of rhizobia with leguminous plants. In both cases, the plant partners provide the symbionts with reduced carbon in exchange for fixed nitrogen (Meeks et al., 1985; Steinberg \& Meeks, 1991; Vance et al., 1998). However, cyanobacteria-plant interactions are primarily unidirectional from plant to cyanobacterial partner (Meeks, 1998), in contrast to the complex signal exchanges between both partners that define the rhizobia-legume plant associations (Oke \& Long, 1999; Schultze \& Kondorosi, 1998).

We are interested in identifying the interactions between the bryophyte hornwort Anthoceros punctatus and N. punctiforme leading to the control of cyanobacterial growth, metabolism and cellular differentiation in the formation of a stable symbiotic association. Analogous to the characterizations of rhizobia-plant associations, the N. punctiforme-A. punctatus interactions can be defined in two stages. In the first, or infective, stage, the symbiotic association is established following invasion of pre-existing cavities in A. punctatus gametophyte tissue by motile hormogonia of $N$. punctiforme. The plant partner both stimulates the formation of hormogonia (Campbell \& Meeks, 1989), and produces signals that affect the chemotactic motility of the hormogonia (Knight \& Adams, 1996). A. punctatus also influences the behaviour of hormogonia through regulation of as yet unidentified genes (Campbell et al., 1998) and activates repressive mechanisms in $\mathrm{N}$. punctiforme that block subsequent hormogonium formation once the cavities have been colonized (Cohen \& Meeks, 1997). The second stage of interaction results in the formation of a functional, or effective, diazotrophic association. The functional symbiotic growth state is characterized by negative regulation of $N$. punctiforme growth and metabolism (Meeks, 1998), and an increase in the frequency of heterocyst differentiation (Enderlin \& Meeks, 1983) and the rate of nitrogen fixation (Steinberg \& Meeks, 1989), coupled with the release of fixed nitrogen as ammonium (Joseph \& Meeks, 1987; Meeks et al., 1985). The enhanced frequency of symbiotic heterocyst differentiation appears to be independent of direct nitrogen control (Campbell \& Meeks, 1992).

These observations imply that there is a set of Nostoc spp. genes that respond to the initial plant signals affecting hormogonium formation and behaviour, leading to the establishment of the cyanobacterial colony in the plant tissue. A second separate set of genes is present in symbiotically competent Nostoc strains and responds to the growth conditions in the plant, resulting in enhanced heterocyst differentiation and nitrogen fixation, as well as other physiological changes in the symbiotic growth state. Therefore, we hypothesize that heterocyst differentiation and nitrogen fixation are dispensable during the initial establishment of cyanobacterial-plant symbiosis. This hypothetical condition is analogous to naturally occurring non-effective rhizobia-legume associations that are more parasitic than mutualistic in nature (Denison, 2000).

In the present study, we characterized three mutant $N$. punctiforme strains unable to differentiate heterocysts $\left(\mathrm{Het}^{-}\right)$and examined their ability to form a symbiotic association with the plant partner A. punctatus. The three mutants have defects in $n t c A$, hetR and hetF. ntcA encodes a DNA-binding protein in the Crp family of bacterial transcriptional regulators. NtcA functions as a nitrogen-dependent global regulator in cyanobacteria and controls the transcription of a number of genes expressed in the entire course of heterocyst development (Herrero et al., 2001; Fiedler et al., 2001). Transcription of het $R$ is indirectly dependent on $n t c A$. het $R$ is temporally the first heterocyst-specific gene induced in the developmental cascade and is considered to be the primary activator of heterocyst differentiation (Wolk, 2000). HetR exhibits a serine-dependent autoprotease activity (Zhou et al., 1998), regulates its own transcription (Black et al., 1993; Buikema \& Haselkorn, 2001), and is sufficient to induce heterocyst differentiation when ectopically expressed under nitrogenreplete conditions (Buikema \& Haselkorn, 2001). Transcription of het $R$ is enhanced in specific cells following combined nitrogen deprivation ( $\mathrm{N}$ step-down), and HetR accumulates in heterocysts (Black et al., 1993; Wong \& Meeks, 2001). HetF is an ancillary positive activator of heterocyst differentiation that functions in the enhanced transcription of het $R$ and the localization of HetR to differentiating cells (Wong \& Meeks, 2001).

The results of this study provide evidence that the differentiation and behaviour of hormogonia are necessary and sufficient in the initial establishment of symbiosis, and that currently known genes essential for the initiation of heterocyst differentiation in the free-living growth state are also required for symbiosis-dependent heterocyst differentiation.

\section{METHODS}

Bacterial cultures and media. Wild-type and mutant strains of N. punctiforme (Table 1) were grown in liquid Allen and Arnon medium diluted fourfold (AA/4) and on solidified AA medium plates as previously described (Cohen et al., 1994). When necessary, the medium was supplemented with $2.5 \mathrm{mM}$ $\mathrm{NH}_{4} \mathrm{Cl}$ buffered with $5 \mathrm{mM}$ MOPS (pH 7-8). Neomycin at $25 \mu \mathrm{g} \mathrm{ml}^{-1}$, ampicillin at $10 \mu \mathrm{g} \mathrm{ml}^{-1}$, and erythromycin at 
Table 1. Bacterial strains and plasmids used in this study

\begin{tabular}{|c|c|c|}
\hline Strain or plasmid & Description & Reference \\
\hline \multicolumn{3}{|l|}{$\begin{array}{l}\text { N. punctiforme } \\
\text { strains }\end{array}$} \\
\hline ATCC 29133 & Wild-type & $\begin{array}{l}\text { Rippka \& Herdman } \\
\text { (1992) }\end{array}$ \\
\hline UCD 415 & het $R:: \operatorname{Tn} 5-1063$ & This study \\
\hline UCD 416 & hetF::Tn5-1063 & Wong \& Meeks (2001) \\
\hline UCD 438 & $\begin{array}{l}\text { Wild-type with pSCR } 30 \text { integrated into the } \\
\text { chromosome; wild-type } n t c A \text { and } n t c A:: \Omega-n p t\end{array}$ & This study \\
\hline UCD 444 & $n t c A:: \Omega-n p t$ & This study \\
\hline UCD 462 & UCD 444 carrying pSCR57 & This study \\
\hline UCD 463 & $\begin{array}{l}\text { Wild-type with pSCR74 integrated into the } \\
\text { chromosome; wild-type hetR and hetR:: Tn5-1063 }\end{array}$ & This study \\
\hline UCD 464 & het $R:: \operatorname{Tn} 5-1063$, reconstructed & This study \\
\hline \multicolumn{3}{|l|}{ E. coli strain } \\
\hline DH5 $\alpha-\mathrm{MCR}$ & $\begin{array}{l}\text { Methylation-dependent restriction-defective } \\
\text { derivative of strain DH } 5 \alpha \text {; used for general cloning }\end{array}$ & Gibco-BRL \\
\hline \multicolumn{3}{|c|}{ ( } \\
\hline $\begin{array}{l}\text { pBluescript } \\
\mathrm{KS}(+) ; \mathrm{pBS}\end{array}$ & Cloning vector; $\mathrm{Ap}^{\mathrm{r}}$ & Stratagene \\
\hline pAM996 & $\begin{array}{l}4.3 \mathrm{~kb} \text { region containing Anabaena } 7120 \text { ntcA in } \\
\mathrm{pBS} ; \mathrm{Ap}^{\mathrm{r}}\end{array}$ & Wei et al. (1993) \\
\hline pRL271 & $\begin{array}{l}\text { Conjugatable non-replicating cyanobacterial vector } \\
\text { containing } s a c B ; \mathrm{Em}^{\mathrm{r}} \mathrm{Cm}^{\mathrm{r}}\end{array}$ & Cai \& Wolk (1990) \\
\hline pSCR202 & $\begin{array}{l}\text { Multicopy replicating plasmid for use in } N . \\
\text { punctiforme and E. coli; } \text { Ap }^{r}\end{array}$ & Summers et al. (1995) \\
\hline pSCR9 & $\Omega-n p t$ cassette source vector; $\mathrm{Nm}^{\mathrm{r}} / \mathrm{Km}^{\mathrm{r}}$ & Cohen \& Meeks (1997) \\
\hline pSCR25 & $\begin{array}{l}\text { Interposon plasmid recovered from EcoRI-digested } \\
\text { genomic DNA of strain UCD } 415 ; \mathrm{Nm}^{\mathrm{r}}\end{array}$ & This study \\
\hline pSCR27 & $\begin{array}{l}4.8 \mathrm{~kb} A c c \mathrm{I} \text { fragment subcloned from cosmid clone } \\
5 \mathrm{E} 11 \text { into pBS hybridized to } n t c A \text { probe; } \mathrm{Ap}^{\mathrm{r}}\end{array}$ & This study \\
\hline pSCR28 & $\begin{array}{l}1.2 \mathrm{~kb} \text { HindIII-EcoRV fragment subcloned from } \\
\text { pSCR27 into pBS containing } n t c A \text { as the only ORF; } \\
\mathrm{Ap}^{\mathrm{r}}\end{array}$ & This study \\
\hline pSCR29 & $\begin{array}{l}\Omega \text { - } n p t \text { cassette ligated into internal } M f e I \text { site of } n t c A \\
\text { in pSCR } 27 ; A^{r}\end{array}$ & This study \\
\hline pSCR30 & $\begin{array}{l}6.6 \mathrm{~kb} \text { SpeI-Xhol insert from pSCR29 ligated into } \\
\text { corresponding sites in pRL271; } \mathrm{Em}^{\mathrm{r}} \mathrm{Cm}^{\mathrm{r}} \mathrm{Nm}^{\mathrm{r}} / \mathrm{Km}^{\mathrm{r}}\end{array}$ & This study \\
\hline pSCR57 & $\begin{array}{l}1 \cdot 2 \mathrm{~kb} \text { SalI-EcoRI insert from pSCR } 28 \text { cloned into } \\
\text { the same sites of pSCR } 202\end{array}$ & This study \\
\hline pSCR74 & $\begin{array}{l}\text { EcoRI-digested tnp-inactivated pSCR25 cloned into } \\
\text { Ecl136II-digested pRL271; } \mathrm{Nm}^{\mathrm{r}} \mathrm{Sm}^{\mathrm{r}} \mathrm{Cm}^{\mathrm{r}} \mathrm{Em}^{\mathrm{r}}\end{array}$ & This study \\
\hline
\end{tabular}

$15 \mu \mathrm{g} \mathrm{ml} \mathrm{m}^{-1}$ were used for the selection and maintenance of recombinant $N$. punctiforme strains.

Escherichia coli strain DH5 $\alpha$-MCR (Table 1) and derivatives were grown in Luria-Bertani (LB) broth supplemented with antibiotics at the following concentrations: kanamycin at $25 \mu \mathrm{g} \mathrm{ml} l^{-1}$; ampicillin at $100 \mu \mathrm{g} \mathrm{ml}^{-1}$; and chloramphenicol at $30 \mu \mathrm{g} \mathrm{ml} \mathrm{m}^{-1}$.

Plant cultures and reconstitution of symbiosis. A. punctatus gametophyte tissue was grown in basal medium (Enderlin \& Meeks, 1983) buffered with $5 \mathrm{mM} \mathrm{MES} \mathrm{(pH} \mathrm{6.4)} \mathrm{and} \mathrm{supple-}$ mented with $0.5 \%(\mathrm{w} / \mathrm{v})$ glucose. To reconstitute the symbiotic associations, A. punctatus tissue was first nitrogen- starved in glucose-supplemented basal medium minus ammonium nitrate for 7-10 d. Mutant and wild-type strains of $N$. punctiforme at a total chlorophyll a content of $60 \mu \mathrm{g}$ were then combined with the nitrogen-starved A. punctatus tissue in the same conditioned medium. The percentage of hormogonium filaments in the population was scored microscopically 24-48 h after addition of cells to the plant tissue and was estimated as one of four levels as previously described (Campbell \& Meeks, 1989): no differentiation (0\%), low (5-15\%), intermediate (40-60\%), and high (90-100\%). Symbiotic colonies were counted with the aid of a dissecting microscope after co-culturing for 2 weeks. For some reconstitution experiments involving the $n t c A$ mutant strain UCD 
444 and the corresponding wild-type control, the conditioned medium was supplemented with $2.5 \mathrm{mM} \mathrm{NH}_{4} \mathrm{Cl}$ after the cocultures were established. To test whether the het $R$ or het $F$ mutant symbiotic colonies established by the first round of infection were viable, the reconstitution medium was supplemented with $15 \mathrm{mM}$ nitrate (equimolar sodium and potassium salt) after the initial 2 week co-culture period. Nitrogenase activity of the symbiotic associations was measured by acetylene reduction assay as previously described (Enderlin \& Meeks, 1983).

Akinete viability assay. To specifically induce akinetes, wildtype and het $R$ mutant strains of $N$. punctiforme cultures were incubated in AA/4 medium minus phosphate for 2 weeks. Akinete differentiation was confirmed by light microscopy. The cultures were then incubated for $10 \mathrm{~d}$ in the dark at $4{ }^{\circ} \mathrm{C}$ without shaking. Filaments of each culture typically shortened to about $10-20$ cells per filament after incubation. Equal numbers of c.f.u. (a c.f.u. being defined as an individual filament) from each culture were plated on solidified AA medium. Plating efficiency was determined by dividing the colonies formed on plates by the original number of c.f.u. Thus, any one of the 10-20 cells in a c.f.u. could give rise to the resulting colony. All growth media in these experiments were supplemented with $15 \mathrm{mM}$ nitrate (equimolar sodium and potassium salt) buffered with $5 \mathrm{mM}$ MOPS ( $\mathrm{pH} 7 \cdot 8$ ).

Molecular biology procedures. Plasmids used in this study are shown in Table 1. Preparations and manipulations of plasmid DNA from E. coli were done using standard methods (Sambrook et al., 1989). Large-scale plasmid purifications from E. coli were carried out using a commercial kit (Qiagen). DNA restriction and modification enzymes were obtained from New England Biolabs or Gibco-BRL and used according to the manufacturers' instructions. Total cyanobacterial genomic and plasmid DNA were isolated as previously described (Cohen et al., 1994). DNA sequencing was performed by contract using ABI Prism Sequencers and the dye termination method. Hybridization probes were labelled with $\left[\alpha-{ }^{32} \mathrm{P}\right] \mathrm{dCTP}$ (Dupont, NEN) using a random priming reaction kit from Gibco-BRL. All PCR amplifications were done using reagents from Amersham Scientific. The probe for N. punctiforme $n t c A$ was generated by PCR using primers PntcA1 (5'GTATTTCGTCAGATGGCGACC-3') and PntcA2 (5'-GGTTTATGCACGGTAATCTTC-3'). DNA hybridization analyses were performed with GeneScreen Plus membrane in $50 \%$ formamide hybridization buffers as previously described (Summers et al., 1995).

Isolation and characterization of hetF and hetR mutant strains. Methods for transposon mutagenesis of N. punctiforme with Tn5-1063 and the isolation and characterization of mutants unable to fix nitrogen in air $\left(\mathrm{Fox}^{-}\right)$were described previously (Cohen et al., 1994). Het ${ }^{-}$mutants were distinguished from the $\mathrm{Fox}^{-}$mutants by microscopically examining strains during combined-nitrogen starvation (N stepdown). Characterization of the hetF mutant strain UCD 416 was reported elsewhere (Wong \& Meeks, 2001). To verify that the phenotype of het $R$ mutant strain UCD 415 was due to the transposition event, the mutation was reconstructed as follows. The transposon in strain UCD 415 together with $3.8 \mathrm{~kb}$ of flanking DNA was recovered from EcoRI-digested genomic DNA and self-ligated to form the plasmid pSCR25. The transposase gene in the interposon plasmid was inactivated by filling in a unique NotI site. The modified plasmid was digested with EcoRI and cloned into the sacB-containing positive selection vector pRL271 yielding pSCR74, which was then transferred by conjugation into wild-type N. punctiforme via tri-parental mating (Cohen et al., 1994). Recombinant strain UCD 463, in which pSCR74 was integrated into the chromosome, was isolated by neomycin selection. Strain UCD 464 , in which pRL271 and the wild-type hetR were eliminated via a second recombination event, was selected for by the presence of neomycin and $5 \%(\mathrm{w} / \mathrm{v})$ sucrose.

Construction and complementation of the ntcA mutant. N. punctiforme ntcA was identified in a $4.8 \mathrm{~kb} A c c \mathrm{I}$ fragment of the genomic cosmid 5E11 (Cohen et al., 1994) by Southern hybrization using a 760 bp probe containing Anabaena sp. strain PCC 7120 ntcA from the plasmid pAM996 (Wei et al., 1993). The fragment was cloned into pBluescript $\mathrm{KS}(+)$ (pBS) to form pSCR27. A strongly hybridizing $1 \cdot 2 \mathrm{~kb}$ EcoRVHindIII fragment of pSCR27 was subcloned into pBS to form pSCR28, whose insert sequence contains the complete $n t c A$ gene. To construct $n t c A$ mutant strain UCD 444, ntcA was inactivated in pSCR 27 by insertion of an omega neomycin phosphotransferase gene cassette $(\Omega-n p t$; from the plasmid pSCR9) into an internal MfeI site to form pSCR29 (Fig. 1). The insert in pSCR29 was excised with SpeI and XhoI and was ligated into XbaI-digested pRL271 to form pSCR30, which was then conjugatively transferred into wild-type N. punctiforme via triparental mating (Cohen et al., 1994). Strain UCD 438 , in which pSCR30 was integrated into the genome, was selected by the presence of neomycin and maintained in medium containing erythromycin and neomycin. Strain UCD 444 , in which pRL271 and the wild-type $n t c A$ were eliminated via a second recombination event, was selected for by the presence of neomycin and $5 \%(\mathrm{w} / \mathrm{v})$ sucrose. Restriction mapping followed by Southern hybridization confirmed elimination of the wild-type gene and replacement with the insertionally inactivated gene (data not shown). To complement the ntcA mutation in trans, the $1.2 \mathrm{~kb}$ insert from pSCR 28 was excised with SalI and EcoRI and ligated to the same sites of the N. punctiforme replicating plasmid pSCR202, yielding pSCR57. N. punctiforme maintains 15 copies of pSCR202-based plasmids per chromosome (Summers et al., 1995). pSCR57 was then transformed by electroporation into strain UCD 444 according to a previously described protocol (Summers et al., 1995), yielding strain UCD 462.

Nucleotide sequence analysis and accession numbers. Protein sequence alignments were carried out using the GAP program in SeqWeb v.1.2 (Genetics Computer Group). Similarity searches were carried out using the BLAST program (Altschul et al., 1997) against the databases available at the National Center for Biotechnology Information website (http:// www.ncbi.nlm.nih.gov/BLAST) and the Joint Genome Institute website (http://www.jgi.doe.gov) for the unfinished N. punctiforme genome sequence. The GenBank accession numbers for the coding regions of $n t c A$, het $R$ and het $F$ are AY038370, AF318069 and AF288130, respectively.

\section{RESULTS AND DISCUSSION}

\section{Phenotypes of hetF and hetR mutant strains in the free-living growth state}

Both hetF (strain UCD 416) and hetR (strain UCD 415) mutant strains of $N$. punctiforme were derived by transposon mutagenesis using Tn5-1063 and selected by screening of colony bleaching upon $\mathrm{N}$ step-down (Fig. 1 and Wong \& Meeks, 2001). Both mutants grew normally as vegetative cell filaments when supplemented with nitrate or ammonium. When deprived of combined nitrogen, neither strain showed morphological evidence for the initiation of heterocyst differentiation. Although 
UCD 444

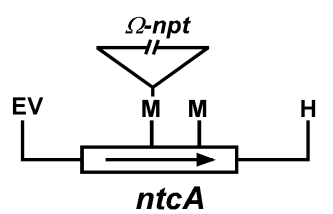

$0.5 \mathrm{~kb}$

\section{UCD 415}

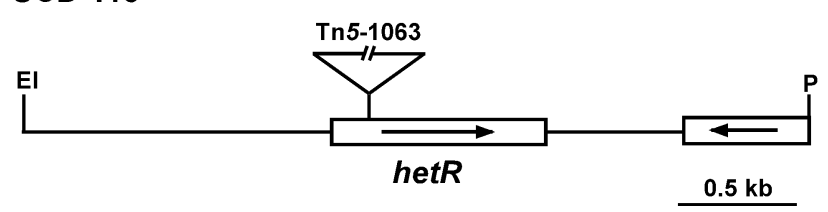

Fig. 1. Physical maps of the $1183 \mathrm{bp}$ region containing ntcA and the $3776 \mathrm{bp}$ region containing hetR. Orientations of the open reading frames are indicated by arrows. The inverted triangles show either Tn5-1063 or $\Omega$-npt insertions as indicated. Selected restriction sites are shown: EcoRI (EI), EcoRV (EV), HindIII (H), Mfel (M) and Pvul (P).

vegetative cells rapidly bleached to a yellow-brown colour, the filaments remained intact (Fig. 2a) and viable for at least 1 month in liquid medium without combined nitrogen, as evidenced by a regreening of the cultures following the addition of combined nitrogen.

Complementation in trans and reconstruction of the hetF mutant was previously reported (Wong \& Meeks, 2001). The hetR mutation in strain UCD 415 was complemented both with the wild-type gene in trans, and with a full-length HetR-green fluorescent protein fusion (data not shown). The N. punctiforme HetR sequence shares $94 \%$ and $88 \%$ identity with those of Nostoc sp. strain PCC 9229 and Anabaena PCC 7120, respectively. The $N$. punctiforme betR gene has similar transcriptional characteristics as Anabaena PCC 7120 hetR (Wong \& Meeks, 2001). The hetR mutation was reconstructed as strain UCD 464, which exhibited the same $\mathrm{Het}^{-}$phenotype as strain UCD 415. All Tn5-1063induced mutants of $N$. punctiforme characterized to date show fidelity to the transposition events (Campbell et al., 1996, 1997; Cohen \& Meeks, 1997; Wong \& Meeks, 2001), in contrast to results with Anabaena PCC 7120 (Ernst et al., 1992; Liang et al., 1992), where secondary mutations and not the transposition events can cause the mutant phenotype.

When starved for phosphate in the presence of nitrate, het $R$ mutant strains UCD 415 and UCD 464 formed large cells similar in overall structure to akinetes, but lacking their characteristic granular appearance (Fig. 2c, d). hetR mutants of Nostoc ellipsosporum were reported to be defective in both heterocyst and akinete differentiation (Léganes et al., 1994), suggesting a common developmental pathway for the two cell types, bifurcating downstream of HetR (Wolk, 2000). Akinetes of $N$. punctiforme are more resistant to prolonged incubation at low temperature than are vegetative cells (Michael Summers, personal communication). To test the chilling resistance of the het $R$ mutant akinete-like cells, exponentially growing wild-type vegetative filaments, wild-type akinetes and strain UCD 464 akinetelike cells were assayed for viability after incubation for $10 \mathrm{~d}$ in the dark at $4{ }^{\circ} \mathrm{C}$. The plating efficiency of the exponential-phase culture was $0 \cdot 1 \%$, while cultures of the wild-type induced akinetes and strain UCD 464
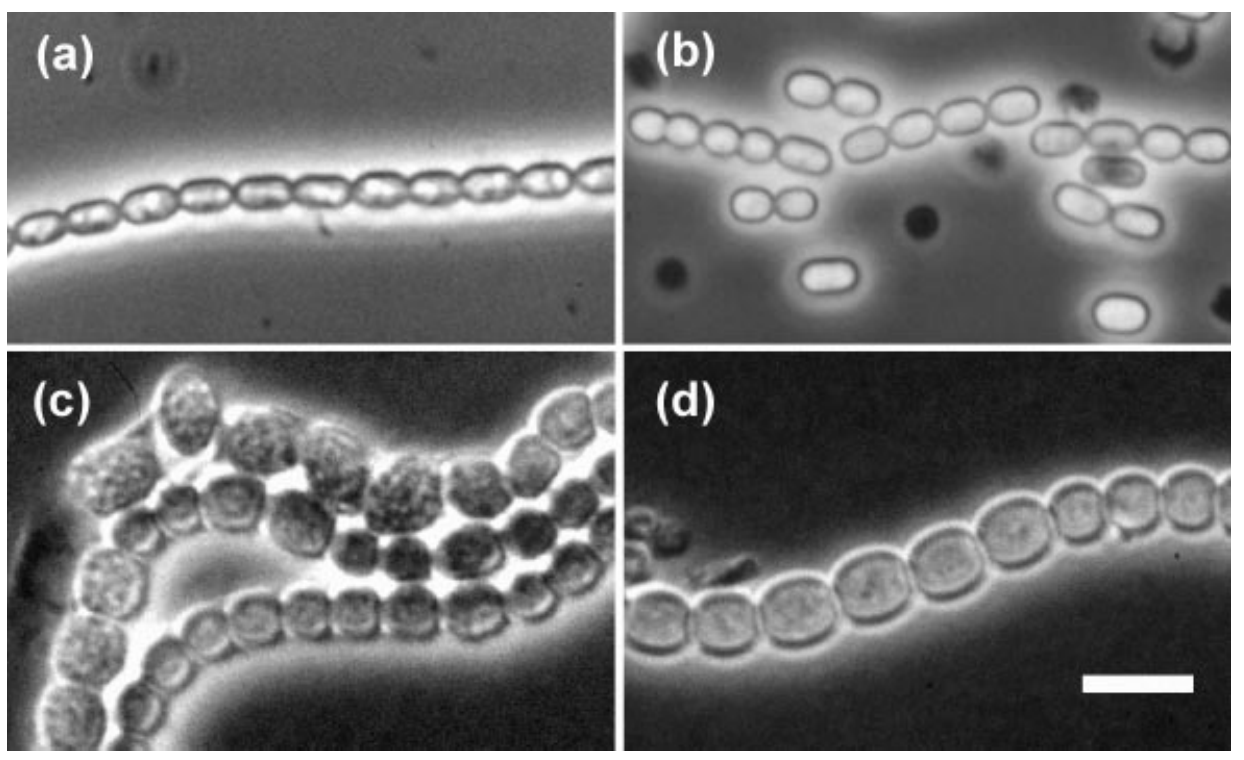

Fig. 2. Photomicrographs of filaments from wild-type and mutant strains of $N$. punctiforme. Intact vegetative cell filament of hetR mutant strain UCD 415 (a) and fragmenting filaments of $n t c A$ mutant strain UCD 444 (b) observed after 36-48 $\mathrm{h}$ in medium free of combined nitrogen. Wild-type akinetes (c) and hetR mutant strain UCD 464 akinete-like cells (d) observed after a 2 week incubation in medium without phosphate. Notice the lack of granular texture in the akinetelike cells in (d) in contrast to (c). Bar, $10 \mu \mathrm{m}$. 
akinete-like cells were $10 \%$ and $9 \cdot 8 \%$, respectively. The increase in viability after low-temperature incubation of the akinete-like cells is essentially identical to that of the wild-type akinetes. We conclude that while HetR may subtly influence the process of akinete development, it is not essential for the initiation of differentiation and certain akinete properties such as chilling resistance. Since only morphology was scored in the N. ellipsosporum het $R$ mutant (Léganes et al., 1994), it is possible that akinete-like cells were not detected because of their morphological resemblance to vegetative cells. An additional possibility is that the differentiation process was delayed in the het $R$ mutant relative to wild-type $N$. ellipsosporum filaments. While akinete formation was not specifically induced, nor chilling resistance tested, in $N$. punctiforme het $F$ and $n t c A$ mutant cultures, akinetelike cells, similar to those of the induced het $R$ mutant culture, were consistently observed in aged cultures.

\section{Phenotype of the ntcA mutant strain in the free-living growth state}

The N. punctiforme ntcA mutant strain UCD 444 was constructed by replacing the wild-type ntcA with one interrupted by an $\Omega-n p t$ cassette (Fig. 1). Similar to Anabaena PCC 7120 ntcA mutants (Frías et al., 1994; Wei et al., 1994), strain UCD 444 failed to grow on nitrate and initiate heterocyst differentiation. These defects are consistent with the loss of a positive global regulator of nitrogen assimilation. Strain UCD 444 carrying multiple copies of $n t c A$ (strain UCD 462) differentiated heterocysts at the wild-type frequency and spacing pattern. This was in contrast to the overproduction of heterocysts that occurred when the copy number of hetF or hetR was elevated in trans (Wong \& Meeks, 2001), and is consistent with the role of NtcA in general nitrogen regulation and not in the specific activation of heterocyst differentiation.

When shifted to medium without ammonium, vegetative cells of strain UCD 444 randomly lysed, so that the filaments became short, debris accumulated in the medium (Fig. 2b), and the culture remained a light green colour. This behaviour is in contrast to the rapid bleaching and non-lytic response of the hetF and het $R$ mutants to nitrogen starvation. A similar non-bleaching and lytic response has been reported for a $n t c A$ mutant strain of the unicellular, non-diazotrophic Synechococcus sp. strain PCC 7942 (Sauer et al., 1999) and may indicate a common signalling pathway shared between chlorosis and induction of heterocyst differentiation.

\section{Symbiotic phenotypes of the hetF and hetR mutants}

hetF mutant strain UCD 416 and hetR mutant strain UCD 464 differentiated an intermediate level (40-60\%) of motile hormogonia in co-cultures with nitrogenstarved A. punctatus tissue, which secretes an as yet unidentified hormogonium-inducing factor (HIF) into the nutrient-limited growth medium (Campbell \& Meeks, 1989). These strains infected A. punctatus tissue
Table 2. Infection frequencies of wild-type and mutant strains of $N$. punctiforme

\begin{tabular}{|lc|}
\hline N. punctiforme strain & Infection frequency* \\
\hline ATCC 29133 (wild-type) & $0 \cdot 28 \pm 0 \cdot 05(5) ; 0 \cdot 13 \pm 0 \cdot 01(2) \dagger$ \\
UCD $416($ hetF) & $0 \cdot 26 \pm 0 \cdot 06(3)$ \\
UCD $464($ het $R)$ & $0 \cdot 36 \pm 0 \cdot 04(4)$ \\
UCD $444(n t c A)$ & $0(3) ; 0(3) \dagger$ \\
UCD $438\left(n t c A^{+}\right)$ & $0 \cdot 36 \pm 0 \cdot 05(3)$ \\
UCD $462\left(n t c A^{+}\right)$ & $0 \cdot 37 \pm 0 \cdot 07(5)$ \\
\hline
\end{tabular}

*Values are the mean number \pm SE of symbiotic colonies per mg dry weight of tissue per unit of $N$. punctiforme cells added (as $\mu \mathrm{g}$ chlorophyll a). Symbioses were reconstituted in the absence of combined nitrogen unless otherwise noted. Number of repetitions is indicated in parentheses.

† Reconstitutions in the presence of $2.5 \mathrm{mM}$ ammonium chloride.

at a frequency similar to that of wild-type N. punctiforme during co-culture in the absence of combined nitrogen (Table 2). However, neither strain supported the $\mathrm{N}_{2}$-dependent growth of the plant partner. The symbiotic colonies, which in the wild-type strain are dark green, remained yellow-brown, and the plant tissue slowly lost pigmentation (Fig. 3a). No nitrogenase activity, as analysed by the acetylene reduction assay, was detected from these bleached colonies (data not shown). Viability of the symbiotic colonies of the two mutants and of A. punctatus could be restored by the addition of $15 \mathrm{mM}$ nitrate, as evidenced by the dark greening of the colonies and the plant tissue (Fig. 3b). These results confirm our hypothesis that the infection of A. punctatus by hormogonia leading to the initial establishment of an association is physiologically uncoupled from the development of a functional diazotrophic association. Whether such non-effective cyanobacterial associations occur in the natural habitat of $A$. punctatus is unknown.

We previously established that N. punctiforme Fox ${ }^{-}$ mutants with defects in the heterocyst wall could infect A. punctatus, fix nitrogen, and support dinitrogendependent growth of the plant partner (Campbell et al., 1996, 1997). A conclusion from those studies was that the oxygen-sensitive mutant phenotype can be physiologically complemented by the nearly anoxic environment of the symbiotic cavity (Campbell \& Meeks, 1992). Nevertheless, heterocyst differentiation, even if defective, remained a prerequisite for symbiotic nitrogenase expression. The results with the het $R$ and het $F$ mutants verify the requirement for heterocyst differentiation in nitrogenase expression (Elhai \& Wolk, 1990). They are also consistent with the lack of a gene cluster encoding a vegetative-cell nitrogenase in the genome sequence of N. punctiforme (http:// www.jgi.doe.gov). Such a vegetative-cell nitrogenase functions under microoxic conditions in some heterocyst-forming cyanobacteria, such as Anabaena variabilis (Thiel et al., 1995). 


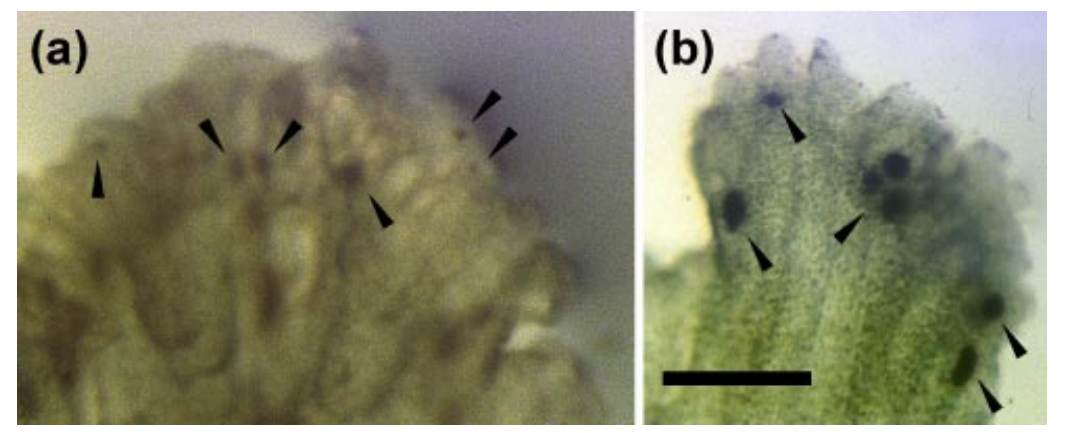

Fig. 3. (a) Symbiotic associations between $N$. punctiforme hetR mutant strain UCD 464 and $A$. punctatus 2 weeks after coculture without combined nitrogen. Bleaching of the $A$. punctatus tissue and symbiotic $N$. punctiforme colonies are evident in this micrograph, which in turn makes it difficult to resolve cellular outlines and chloroplasts. (b) Tissue from the same culture after 2 weeks of growth supplemented with $15 \mathrm{mM}$ nitrate. Note the distinct chloroplasts in $A$. punctatus cells in (b), in contrast to (a). Arrowheads indicate colonies in symbiotic cavities. Bar, $0.5 \mathrm{~mm}$.

The nitrogen-independent enhanced frequency of heterocysts observed in symbiosis is hypothetically a consequence of a symbiosis-specific signalling system responding to plant-produced signals (Campbell \& Meeks, 1992). This system is predicted to supersede the nitrogen signalling system which responds to nitrogen limitation and initiates heterocyst differentiation in the free-living growth state (Meeks, 1998). The nature of the symbiotic signal and where it might enter the developmental cascade is unknown. Based on the results with the het $R$ and hetF mutants, both free-living and symbiotic heterocyst differentiation share a common developmental pathway. Because a functional NtcA is required for the transcription of genes expressed throughout heterocyst development (Herrero et al., 2001; Fiedler et al., 2001), we speculate that the symbiotic signal should enter the developmental cascade at or prior to $n t c A$. Presently, no genes nor enzyme activities are known to be involved in the initiation of heterocyst differentiation cascade prior to $n t c A$.

\section{Symbiotic phenotype of the ntcA mutant}

In contrast to the het $F$ and het $R$ mutants, ntc $A$ mutant strain UCD 444 responded with markedly less intensity than the wild-type in the differentiation of hormogonia in co-cultures with A. punctatus. The low level (5-15\%) of hormogonia formed had the typical morphology and gliding properties of wild-type hormogonia. However, strain UCD 444 failed to infect A. punctatus tissue in multiple trials of co-cultures in the absence or presence of $2.5 \mathrm{mM}$ ammonium chloride (Table 2). Ammonium was added periodically during these experiments to ensure viability of the $n t c A$ mutant and increase the probability of an infection event. Such a supplementation was not necessary with the infective het $R$ and hetF mutant strains. Epiphytic growth of strain UCD 444 was present on the gametophyte tissue at the end of the 2 week co-culture period, indicating that the ammonium level was sufficient to maintain viability. Sufficient time (up to 1 month) was allowed in the co- culture experiments with strain UCD 444 that any delayed infection and formation of small colonies in the absence or presence of ammonium would have been detected microscopically. When ammonium was not included in the co-cultures, strain UCD 444 lysed as it did in nitrogen-limited culture in the absence of $A$. punctatus. Wild-type Nostoc strains, including N. punctiforme, can infect $A$. punctatus in the presence of 1-5 mM ammonium, but at a lower frequency (Table 2; Enderlin \& Meeks, 1983). The colonies established in the presence of ammonium are small and have low nitrogenase activity.

The non-infective phenotype of strain UCD 444 was complemented by the presence of one or more copies of $n t c A$. Both strain UCD 438, which carries a wild-type and a mutant copy of $n t c A$ in the genome, and strain UCD 462, which is strain UCD 444 carrying a wild-type copy of $n t c A$ on a multicopy replicating plasmid (pSCR57), yielded wild-type infection frequencies and supported growth of the A. punctatus association in the absence of combined nitrogen (Table 2). Thus, the noninfective phenotype of strain UCD 444 can be ascribed to the mutation of $n t c A$ and not to an unknown and undetected genetic lesion. However, the lack of infection by the $n t c A$ mutant precludes any attempts to define a regulatory role for NtcA in the enhanced frequency of symbiotic heterocyst differentiation.

The infection-defective phenotype of $n t c A$ mutant strain UCD 444 was unexpected. NtcA is a relatively wellcharacterized global nitrogen regulator in cyanobacteria (Herrero et al., 2001). There is no previous evidence suggesting that hormogonium development is regulated by nitrogen control. Hormogonium differentiation induced by HIF is insensitive to the absence or presence of ammonium (Campbell \& Meeks, 1989), and hormogonia will infect $A$. punctatus in the presence of nitrate or ammonium (Table 2; Enderlin \& Meeks, 1983). Therefore, the reduced frequency of hormogonium differentiation in strain UCD 444 does not extrapolate to the failure of the formed hormogonia to infect $A$. 
punctatus; the expected number of infections would be lower, but should still be greater than zero. Nevertheless, the results establish a connection between a functional copy of $n t c A$, the extent of hormogonium differentiation and the behaviour of the formed hormogonia.

There is precedence for both low and highly infective hormogonia from natural Nostoc isolates and mutant strains. Nostoc sp. strain ATCC 27896 (PCC 6320) converts essentially $100 \%$ of its filaments into motile hormogonia in the presence of HIF, but the hormogonia have a low and delayed infection frequency of $A$. punctatus (Meeks, 1998). When reisolated from $A$. punctatus tissue and again co-cultured, the same rare, delayed infection is observed. Thus, defective infection appears to be a genetic property of the strain, perhaps resulting from insensitivity to chemoattractant signals. Conversely, mutation of a gene encoding an alternative group 2 sigma subunit of RNA polymerase $(\operatorname{sig} H)$ in $\mathrm{N}$. punctiforme results in a strain that has a sixfold higher infection frequency of $A$. punctatus than the wild-type (Campbell et al., 1998). The sigH mutant does not differentiate more hormogonia, rather it is the behaviour of the hormogonia that appears to have changed. Collectively, these results indicate that hormogonia differentiation and behaviour in infection of $A$. punctatus tissue involves more complex regulatory circuits than is superficially apparent. Moreover, NtcA appears to be involved in those circuits, suggesting that NtcA may have regulatory roles apart from nitrogen assimilation.

\section{ACKNOWLEDGEMENTS}

This work was supported by grants NRICGP 98-35305-6748 from the United States Department of Agriculture and IBN 0080805 from the United States National Science Foundation. We thank Elsie Campbell and Kari Hagen for a critical review of the manuscript. We thank Jim Golden for providing the pAM996 plasmid.

\section{REFERENCES}

Adams, D. G. \& Duggan, P. S. (1999). Heterocyst and akinete differentiation in cyanobacteria. New Phytol 144, 3-33.

Altschul, S. F., Madden, T. L., Schaffer, A. A., Zhang, J., Zhang, Z., Miller, W. \& Lipman, D. J. (1997). Gapped BLAST and PSI-BLAST: a new generation of protein database search programs. Nucleic Acids Res 25, 3389-3402.

Black, T. A., Cai, Y. \& Wolk, C. P. (1993). Spatial expression and autoregulation of het $R$, a gene involved in the control of heterocyst development in Anabaena. Mol Microbiol 9, 77-84.

Buikema, W. J. \& Haselkorn, R. (2001). Expression of the Anabaena het $R$ gene from a copper-regulated promoter leads to heterocyst differentiation under repressing conditions. Proc Natl Acad Sci U S A 98, 2729-2734.

Cai, Y. P. \& Wolk, C. P. (1990). Use of a conditionally lethal gene in Anabaena sp. strain PCC 7120 to select for double recombinants and to entrap insertion sequences. J Bacteriol 172, 3138-3145.

Campbell, E. L. \& Meeks, J. C. (1989). Characteristics of hormogonia formation by symbiotic Nostoc spp. in response to the presence of Anthoceros punctatus or its extracellular products. Appl Environ Microbiol 55, 125-131.

Campbell, E. L. \& Meeks, J. C. (1992). Evidence for plant-mediated regulation of nitrogenase expression in the Anthoceros-Nostoc symbiotic association. J Gen Microbiol 138, 473-480.

Campbell, E. L., Hagen, K. D., Cohen, M. F., Summers, M. L. \& Meeks, J. C. (1996). The $\operatorname{dev} R$ gene product is characteristic of receivers of two-component regulatory systems and is essential for heterocyst development in the filamentous cyanobacterium Nostoc sp. strain ATCC 29133. J Bacteriol 178, 2037-2043.

Campbell, E. L., Cohen, M. F. \& Meeks, J. C. (1997). A polyketidesynthase-like gene is involved in the synthesis of heterocyst glycolipids in Nostoc punctiforme strain ATCC 29133. Arch Microbiol 167, 251-258.

Campbell, E. L., Brahamsha, B. \& Meeks, J. C. (1998). Mutation of an alternative sigma factor in the cyanobacterium Nostoc punctiforme results in increased infection of its symbiotic plant partner, Anthoceros punctatus. J Bacteriol 180, 4938-4941.

Castenholz, R. W. \& Waterbury, J. B. (1989). Oxygenic photosynthetic bacteria (section 19), group 1. Cyanobacteria. In Bergey's Manual of Systematic Bacteriology, vol. 3, pp. 17101728. Edited by J. T. Staley, M. P. Bryant, N. Pfenning \& J. G. Holt. Baltimore: Williams \& Wilkins.

Cohen, M. F. \& Meeks, J. C. (1997). A hormogonium regulating locus, hrmUA, of the cyanobacterium Nostoc punctiforme strain ATCC 29133 and its response to an extract of a symbiotic plant partner Anthoceros punctatus. Mol Plant-Microbe Interact 10, 280-289.

Cohen, M. F., Wallis, J. G., Campbell, E. L. \& Meeks, J. C. (1994). Transposon mutagenesis of Nostoc sp. strain ATCC 29133, a filamentous cyanobacterium with multiple cellular differentiation alternatives. Microbiology 140, 3233-3240.

Denison, R. F. (2000). Legume sanctions and the evolution of symbiotic cooperation by rhizobia. Am Nat 156, 567-576.

Elhai, J. \& Wolk, C. P. (1990). Developmental regulation and spatial pattern of expression of the structural genes for nitrogenase in the cyanobacterium Anabaena. EMBO J 9, 3379-3388.

Enderlin, C. S. \& Meeks, J. C. (1983). Pure culture and reconstitution of the Anthoceros-Nostoc symbiotic association. Planta 158, 157-165.

Ernst, A., Black, T., Cai, Y., Panoff, J. M., Tiwari, D. N. \& Wolk, C. P. (1992). Synthesis of nitrogenase in mutants of the cyanobacterium Anabaena sp. strain PCC 7120 affected in heterocyst development or metabolism. J Bacteriol 174, 6025-6032.

Fiedler, G., Muro-Pastor, A., Flores, E. \& Maldener, I. (2001). NtcA-dependent expression of the $\operatorname{dev} B C A$ operon, encoding a heterocyst-specific ATP-binding cassette transporter in Anabaena spp. J Bacteriol 183, 3795-3799.

Frías, J. E., Flores, E. \& Herrero, A. (1994). Requirement of the regulatory protein NtcA for the expression of nitrogen assimilation and heterocyst development genes in the cyanobacterium Anabaena sp. PCC 7120. Mol Microbiol 14, 823-832.

Herrero, A., Muro-Pastor, A. M. \& Flores, E. (2001). Nitrogen control in cyanobacteria. J Bacteriol 183, 411-425.

Joseph, C. M. \& Meeks, J. C. (1987). Regulation of expression of glutamine synthetase in a symbiotic Nostoc strain associated with Anthoceros punctatus. J Bacteriol 169, 2471-2475.

Knight, C. D. \& Adams, D. G. (1996). A method for studying chemotaxis in nitrogen fixing cyanobacterium-plant symbiosis. Physiol Mol Plant Pathol 49, 73-77.

Leganés, F., Fernández-Piñas, F. \& Wolk, C. P. (1994). Two mutations that block heterocyst differentiation have different 
effects on akinete differentiation in Nostoc ellipsosporum. Mol Microbiol 12, 679-684.

Liang, J., Scappino, L. \& Haselkorn, R. (1992). The patA gene product, which contains a region similar to CheY of Escherichia coli, controls heterocyst pattern formation in the cyanobacterium Anabaena 7120. Proc Natl Acad Sci US A 89, 5655-5659.

Meeks, J. C. (1998). Symbiosis between nitrogen-fixing cyanobacteria and plants. BioScience 48, 266-276.

Meeks, J. C., Enderlin, C. S., Joseph, C. M., Chapman, J. S. \& Lollar, M. W. (1985). Fixation of $\left[{ }^{13} \mathrm{~N}\right] \mathrm{N}_{2}$ and transfer of fixed nitrogen in the Anthoceros-Nostoc symbiotic association. Planta 164, 406-414.

Oke, V. \& Long, S. R. (1999). Bacteroid formation in the Rhizobium-legume symbiosis. Curr Opin Microbiol 2, 641-646.

Rippka, R. \& Herdman, M. (1992). In Pasteur Culture Collection of Cyanobacteria in Axenic Culture, pp. 44-57. Paris: Institut Pasteur.

Sambrook, J., Maniatis, T. \& Fritsch, E. F. (1989). Molecular Cloning: a Laboratory Manual, 2nd edn. Cold Spring Harbor, NY : Cold Spring Harbor Laboratory.

Sauer, J., Görl, M. \& Forchhammer, K. (1999). Nitrogen starvation in Synechococcus PCC 7942: involvement of glutamine synthetase and NtcA in phycobiliprotein degradation and survival. Arch Microbiol 172, 247-255.

Schultze, M. \& Kondorosi, A. (1998). Regulation of symbiotic root nodule development. Annu Rev Genet 32, 33-57.

Steinberg, N. A. \& Meeks, J. C. (1989). Photosynthetic $\mathrm{CO}_{2}$ fixation and ribulose bisphosphate carboxylase/oxygenase activity of Nostoc sp. strain UCD 7801 in symbiotic association with Anthoceros punctatus. J Bacteriol 171, 6227-6233.

Steinberg, N. A. \& Meeks, J. C. (1991). Physiological sources of reductant for nitrogen fixation activity in Nostoc sp. strain UCD 7801 in symbiotic association with Anthoceros punctatus. J Bacteriol 173, 7324-7329.

Summers, M. L., Wallis, J. G., Campbell, E. L. \& Meeks, J. C. (1995). Genetic evidence of a major role for glucose-6-phosphate dehydrogenase in nitrogen fixation and dark growth of the cyanobacterium Nostoc sp. strain ATCC 29133. J Bacteriol 177, 6184-6194.
Tandeau de Marsac, N. (1994). Differentiation of hormogonia and relationships with other biological processes. In The Molecular Biology of Cyanobacteria, pp. 825-842. Edited by D. A. Bryant. Dordrecht: Kluwer.

Thiel, T., Lyons, E. M., Erker, J. C. \& Ernst, A. (1995). A second nitrogenase in vegetative cells of a heterocyst-forming cyanobacterium. Proc Natl Acad Sci US A 92, 9358-9362.

Vance, C. P., Miller, S. S., Driscoll, B. T., Robinson, D. L., Trepp, G., Gantt, J. S. \& Samas, D. A. (1998). Nodule carbon metabolism: organic acids for $\mathrm{N}_{2}$ fixation. In Biological Nitrogen Fixation for the 21st Century, pp. 443-448. Edited by C. Elmerich, A. Kondorosi \& W. E. Newton. Dordrecht: Kluwer.

Wei, T. F., Ramasubramanian, T. S., Pu, F. \& Golden, J. W. (1993). Anabaena sp. strain PCC 7120 bifA gene encoding a sequencespecific DNA-binding protein cloned by in vivo transcriptional interference selection. J Bacteriol 175, 4025-4035.

Wei, T. F., Ramasubramanian, T. S. \& Golden, J. W. (1994). Anabaena sp. strain PCC 7120 ntcA gene required for growth on nitrate and heterocyst development. J Bacteriol 176, 4473-4482.

Wolk, C. P. (2000). Heterocyst formation in Anabaena. In Prokaryotic Development, pp. 83-104. Edited by Y. V. Brun \& L. J. Shimkets. Washington, DC: American Society for Microbiology.

Wolk, C. P., Ernst, A. \& Elhai, J. (1994). Heterocyst metabolism and development. In The Molecular Biology of Cyanobacteria, pp. 769-823. Edited by D. A. Bryant. Dordrecht: Kluwer Academic Publishers.

Wong, F. C. \& Meeks, J. C. (2001). The hetF gene product is essential to heterocyst differentiation and affects HetR function in the cyanobacterium Nostoc punctiforme. J Bacteriol 183, 26542661.

Zhou, R., Wei, X., Jiang, N., Li, H., Dong, Y., Hsi, K. L. \& Zhao, J. (1998). Evidence that HetR protein is an unusual serine-type protease. Proc Natl Acad Sci US A 95, 4959-4963.

Received 13 July 2001; revised 30 August 2001; accepted 12 September 2001. 